\title{
ON THE PROFILES AND THE POLARIZATION OF RAMAN SCATTERED EMISSION LINES IN THE SYMBIOTIC STARS: II
}

\author{
K.W. LEE \\ Kyungpook National Univ., Taegu 702-701, South KOREA \\ kwlee@vega.kyungpook.ac.kr \\ AND \\ H.-W. LEE \\ Seoul National Univ., Seoul 151-742, South KOREA \\ hwlee@astro9.snu.ac.kr
}

A Monte Carlo code is used to calculate the profiles and the polarization of Raman scattered O VI lines $(\lambda \lambda 6827,7088)$ in symbiotic stars. Various wind models around the cool giant are investigated including disk type and bipolar wind models. We obtain a large degree of polarization in the blue part and the red-asymmetric profile, which is generic to the Raman scattered feature in symbiotic stars.

We also investigate the effect of the orbital motion of the hot component around the cool giant, where the orbital velocity is comparable to the wind terminal velocity. In the case of small inclination angle of the orbital plane, we obtain the polarization flip around the red wing of the scattered feature and the triple peak structure in the polarized flux, which is characteristic of many symbiotic systems including RR Tel.

Brief observational consequences are discussed and it is concluded that spectropolarimetry may provide a powerful diagnostic about the physical conditions of symbiotic stars. 